\title{
A hand full of numbers: a role for offloading in arithmetics learning?
}

\section{Annelise Júlio Costa ${ }^{1}$, Júlia Beatriz Lopes Silva ${ }^{1,2}$, Pedro Pinheiro Chagas ${ }^{1,3}$, Helga Krinzinger ${ }^{4}$, Jan Lonneman ${ }^{5,6,7}$, Klaus Willmes ${ }^{5}$, Guilherme Wood ${ }^{8}$ and Vitor Geraldi Haase ${ }^{1,2,3}$}

1 Developmental Neuropsychology Laboratory, Department of Psychology, Universidade Federal de Minas Gerais, Belo Horizonte, Minas Gerais, Brazil

2 Programa de Pós-graduação em Saúde da Criança e do Adolescente, Faculdade de Medicina, Universidade Federal de Minas Gerais, Belo Horizonte, Minas Gerais, Brazil

3 Programa de Pós-graduação em Neurociências, Universidade Federal de Minas Gerais, Belo Horizonte, Minas Gerais, Brazil

${ }^{4}$ Section Child Neuropsychology, Department of Child and Adolescent Psychiatry, RWTH Aachen University, Aachen, Germany

${ }^{5}$ Section Neuropsychology, Department of Neurology, RWTH Aachen University, Aachen, Germany

${ }^{6}$ Department of Educational Psychology, Institute for Psychology, Goethe University Frankfurt am Main, Frankfurt am Main, Germany

7 Center for Individual Development and Adaptive Education of Children at Risk, Frankfurt am Main, Germany

${ }^{8}$ Department of Neuropsychology, Institute of Psychology, Karl-Franzens University of Graz, Graz, Austria

\section{Edited by:}

Frank Domahs, RWTH Aachen

University Hospital, Germany

Reviewed by:

Virginie Crollen, Université Catholique

de Louvain, Belgium

Robert Reeve, University of

Melbourne, Australia

${ }^{*}$ Correspondence:

Annelise Júlio Costa, Developmental Neuropsychology Laboratory,

Department of Psychology,

Universidade Federal de Minas

Gerais, Avenida Antônio Carlos, 6627,

31270-901 Belo Horizonte, Minas

Gerais, Brazil.

e-mail: annelisejulio@gmail.com
Finger counting has been associated to arithmetic learning in children. We examined children with $(n=14)$ and without $(n=84)$ mathematics learning difficulties with ages between 8 and 11 years. Deficits in finger gnosia were found in association to mathematical difficulties. Finger gnosia was particularly relevant for the performance in word problems requiring active manipulation of small magnitudes in the range between 1 and 10. Moreover, the deficits in finger gnosia could not be attributed to a shortage in working memory capacity but rather to a specific inability to use fingers to transiently represent magnitudes, tagging to be counted objects, and reducing the cognitive load necessary to solve arithmetic problems. Since finger gnosia was more related to symbolic than to non-symbolic magnitude processing, finger-related representation of magnitude seems to be an important link for learning the mapping of analog onto discrete symbolic magnitudes.

Keywords: finger gnosia, mathematics difficulties, number sense, dyscalculia

\section{INTRODUCTION}

Finger counting is frequently used by children, and under some circumstances by adults too. This ability seems to be spontaneously learned, and practice varies widely across cultures (Domahs et al., 2010), with some cultures explicitly teaching this strategy (Guha, 2006). Finger counting is especially important in children beginning to learn arithmetics, being influenced by the socio-cultural background. Jordan et al. (2008) observed that while middleclass children resorted to this strategy from the first grade on and used it progressively less after the second grade, children of lower socio-economic strata began later to use fingers for counting and persisted to do so for a more extended period of time. Moreover, visual input and imitation play important roles in finger counting, as congenitally blind children engage less frequently in this practice, and use fingers in culturally non-canonical ways (Crollen et al., 2011a). Along with other less efficient strategies, finger counting is a classical resource employed by children with difficulties in learning arithmetics, which suggests difficulties with facts learning (Butterworth, 1999; Geary et al., 2000).

Usually, children start to count on fingers at the age of 3 years, typically persisting until the beginning of second grade (Lecointre et al., 2005). Alibali and DiRusso (1999) investigated the role of gesture on the development of counting abilities in 4-year-old children. Analysis of the error patterns committed in different counting conditions revealed that finger counting helps children to improve two aspects of one-to-one correspondence principle: keeping track of the counted objects and coordinating the number words with the objects. They proposed that finger counting serves as an offloading mechanism to reduce cognitive demands by physically instantiating some contents of working memory.

In a recent review, Raghubar et al. (2010) reported in detail the role of working memory in math achievement. A robust effect of verbal working memory on math performance has been consistently found in the literature, particularly regarding numeric stimuli (i.e., digit span and counting span). In contrast, concerning visuospatial working memory (i.e., Corsi Blocks) the picture has proved to be less consistent. General studies have shown that the central executive component plays a major role. Swanson (2004) has shown that effects of the slave systems in the multicomponential working memory model are attenuated when analyses include central executive measures. Hecht et al. (2001) observed that phonological decoding assessed by means of the phoneme deletion task is longitudinally predictive of mathematics achievement, but, performance on this task imposes demands on working memory resources. Direct implication of central executive mechanisms in math learning difficulties has been demonstrated several 
times (e.g., Bull and Scerif, 2001; van der Sluis et al., 2004, see review in Raghubar et al., 2010).

The signature of finger counting is observed also in the error patterns of children learning arithmetics. Domahs et al. (2008) observed that arithmetic errors committed by children beginning to learn fact retrieval frequently deviate from the correct result by multiples of five, suggesting that a sub-base five, probably related to the hand structure, plays an important role in calculation procedures. Later findings by Domahs et al. (2010) disclosed that the comparison of Arabic number symbols in adults is also influenced by a sub-base five system inherent in culturally bound finger counting habits. Besides, experimental studies with adults showed that number processing interacts in complex ways with egocentric, finger-based, and allocentric spatial representations, being modulated by finger counting habits (Fischer, 2008; Conson et al., 2009; Di Luca et al., 2010). Other results indicate that, both in a parity judgment (Sato et al., 2007) as well as in a counting task (Andres et al., 2007), motor evoked potentials for right hand muscles are modulated by number magnitude. These results are suggestive of a special role of embodied representations in the development of cognitive processes, finger representations being specific to the number and arithmetic domain.

Proficiency in finger counting relies at least to some degree upon the ability to locate, name, and discriminate individual fingers (i.e., finger gnosia). Accordingly, Gracia-Bafalluy and Noël (2008) showed that children with deficits in finger discrimination abilities also present a deficit in enumeration and counting tasks in comparison to children with above average finger discrimination abilities. A first longitudinal study by Fayol et al. (1998) found that a composite score encompassing finger gnosia and other somatosensory relatively complex abilities assessed at 5 years of age was able to predict math performance 1 year later $(r=0.46)$. These results were confirmed in a subsequent study for a period of 3 years of observation (Marinthe et al., 2001). However, these findings should be seen in perspective because math performance also correlated to more general developmental tests $(r=0.44)$.

Using more specific measures of finger gnosia, Noël (2005) also observed an association of finger recognition and discrimination at the beginning of first grade with mathematics performance 15 months later. In this study, both the specificity and relative predictive power of finger gnosia on math achievement were examined. Finger gnosia correlated with mathematics performance $(r=0.48)$, but not with reading achievement $(r=0.11)$. Performance on the WISC Coding task also correlated with math achievement, but to a lower degree ( $r=-0.21)$. Moreover, $46 \%$ of variance in second grade mathematics achievement was explained by beginning first grade measures of finger gnosia as well as second grade measures of handwriting and block design. This suggests that the association between finger gnosia and mathematics achievement is both strong and functionally specific. Similar results regarding the role of finger gnosia in number system knowledge and calculation skills were obtained by Penner-Wilger et al. (2007) in first grade children. A role for finger gnosia in learning arithmetics was also inferred from a training study conducted by Gracia-Bafalluy and Noël (2008). However, their experimental design was based on extreme performance groups, what renders the results subject to a regression to the mean interpretation
(Fischer, 2010). These data suggest that finger counting related to gnosia abilities plays an important role in arithmetic learning in beginning formal schooling. One important aspect of this question is the investigation of the shared neurocognitive correlates of finger gnosia, number processing, and arithmetics.

The association between finger counting and mathematics performance has been traditionally interpreted in the context of the syndrome discovered by Gerstmann in 1924, and which carries his name (Gerstmann, 1940; see also Rusconi et al., 2010). In a series of case analyses, Gerstmann observed that patients with lesions in the region of the left angular gyrus presented disorders of right-left orientation, dysgraphia, acalculia, and finger agnosia. Gerstmann postulated a deficit in a more basic underlying function. As the internal correlations of the syndrome components are usually lower than their individual correlations with other neuropsychological deficits, the very existence of the syndrome has been subject to heated debate (Rusconi et al., 2010), and the identification of its underlying Grundstörung has eluded research efforts.

Interestingly, mathematical learning difficulties in some children are associated with the other three components of the Gerstmann syndrome (Kinsbourne and Warrington, 1963). However, as children with the so-called developmental Gerstmann syndrome usually exhibit more pronounced evidence of right hemisphere dysfunction, such as lower levels of finger gnosia performance with the left hand, this disorder is nowadays discussed under the rubric of "non-verbal learning disability" (henceforth NLD), and at least one prominent model attributes the underlying dysfunction to white matter damage (Rourke, 1989).

Two main hypotheses have been considered to explain the neural correlates of the relationship between finger gnosia/counting and arithmetic learning and its disorders from a developmental perspective, the localizationist and the functionalist views (Noël, 2005; Crollen et al., 2011b), to which a third hypothesis of redeployment of finger representations (PennerWilger and Anderson, 2008) or neuronal recycling (Dehaene and Cohen, 2007) must be added. According to the localizationist hypothesis, co-occurrence of deficits in finger gnosia and numerical and arithmetical disorders, such as observed in the Gerstmann syndrome, is merely accidental, reflecting topographic vicinity of unrelated functions. Recent evidence compatible with the localizationist hypothesis was obtained in adults by structural MRI, confirming that fibers connecting cortical areas related to the Gerstmann tetrad are densely packed beneath the angular gyrus (Rusconi et al., 2009). These authors were, however, unable to trace functional connections between those areas. From another point of view, the functionalist and redeployment hypotheses assume that finger representations are recruited or exaptated for counting because they are computationally suitable to implement number representations required for counting and arithmetic facts (Penner-Wilger and Anderson, 2008).

The only formal empirical test of the localizationist vs. functionalist hypotheses in developing individuals was conducted by Noël (2005). As predicted by the localizationist hypothesis, mathematical achievement was significantly correlated to other components of the Gerstmann constellation, besides finger gnosia: rightleft orientation $(r=-0.34)$, constructional abilities $(r=0.44)$, 
and handwriting $(r=0.43)$. The author planned to test the functionalist hypothesis by contrasting correlations between finger gnosia and performance in arithmetic tasks that are dependent on (i.e., addition) and independent of (i.e., magnitude comparison) finger counting. This latter test failed because number-related performance was explained by a single factor, and because finger gnosia correlated significantly to both kinds of numerical tests (all $r$ s around $0.36-0.38$ ). The negative results regarding a dissociation between number processes which are more or less independent of finger gnosia described by Noël (2005) may lay to a large extent on the methods used to investigate them. The sample size $n=45$ examined in Noël (2005) is too small to reliably distinguish between the existence of one or two latent factors (MacCallum et al., 1999). Moreover, it is unrealistic to expect that the two factors describing two different aspects of number processing would be completely independent of each other. Considering the nature of these processes it is much more reasonable to expect that the factors would be at least moderately correlated. For these reasons, a selective impact of finger gnosia on specific numeric abilities remains elusive in this study. Hence, behavioral studies have established the importance of finger counting on arithmetic learning.

As already pointed out by Gerstmann (1940), brain regions responsible for finger gnosia and arithmetic abilities are neuroanatomic neighbors. A structural neuroimaging study corroborates the importance of both cortical and subcortical structures of the posterior right hemisphere in number processing and calculation. Rykhlevskaia et al. (2009) compared brain structural characteristics of children with dyscalculia to those of typically developing controls. Volumetric analyses revealed reductions of both cortical and gray matter around the inferior parietal sulcus and superior parietal lobule bilaterally. Fractional anisotropy was most altered in the right parietal lobe and tractographic analyses revealed that long range connections between the right fusiform gyrus and temporal-parietal regions via the inferior longitudinal fasciculus were compromised in children with dyscalculia.

fMRI studies have investigated the neural correlates of nonsymbolic magnitude processing and finger representations (Kaufmann et al., 2008). When judging whether the number of fingers presented is smaller or larger than 5, adults show activation of the classical intraparietal areas related to non-symbolic magnitude processing while children also activated more anterior areas of the right intraparietal sulcus and post and precentral sulcus, which are related to hand functions. Moreover, in the same task, children with mathematical difficulties (MD) activate more the left intraparietal cortex, probably as a compensatory strategy (Kaufmann et al., 2009). Interestingly, a recent meta-analysis investigating the developmental fMRI studies of typical and atypical number processing revealed that (intra)parietal activations of dyscalculic children were more anterior than those displayed by controls, suggesting that those children strongly rely on fingerbased number representations (Kaufmann et al., 2011). This suggests that neural impairments in mathematical learning disabilities are related to dysfunctions in a complex network of left and right hemispheric cortical and subcortical structures which typically connect finger counting, discrete magnitudes, and verbal and Arabic representations.
In the present study, the impact of finger gnosia on mathematical abilities was investigated in children with and without MD. In previous studies, an association between finger gnosia and mathematical abilities has been shown (Noël, 2005; Penner-Wilger et al., 2007). Moreover, according to Marinthe et al. (2001) finger gnosia predicted arithmetics achievement. Furthermore, GraciaBafalluy and Noël (2008) reported that finger gnosia training may improve arithmetics achievement. So firstly, it is reasonable to hypothesize that children with MD will present lower finger discrimination abilities in comparison to typically achieving children (TA). Additionally, since previous studies have found morphological and functional interhemispheric differences between TA and MD predominantly in the right hemisphere (Kaufmann et al., 2009; Rykhlevskaia et al., 2009), we may expect that difficulties in finger gnosia should be more pronounced in the (non-dominant) left hand. Secondly, finger discrimination deficits should not be related to more basic aspects of neurologic maturation, such as motor dexterity, and should not be explained by more general cognitive deficits such as working memory or intelligence alone. Thirdly, based on previous findings by Noël (2005), we expect a stronger impact of finger gnosia deficits on counting dependent procedures (i.e., arithmetic word problems) than on tasks tapping the approximate number system (ANS; i.e., magnitude comparison) or fact retrieval (i.e., multiplication facts). Finally, if the localizationist hypothesis is correct, then we should expect moderate to high correlations between finger gnosia and the other functions related to the Gerstmann syndrome constellation, such as right-left orientation and constructional abilities.

\section{MATERIALS AND METHODS PARTICIPANTS}

The study was approved by the local research ethics committee (COEP-UFMG). Children participated only after informed consent was obtained in written form from parents, and orally from children. They were recruited in two distinct phases: in the screening phase, we selected public and private schools from Belo Horizonte and Mariana, Brazil, and used the arithmetics and spelling subtests of the Brazilian School Achievement Test (Teste do Desempenho Escolar, TDE; Stein, 1994). In this phase, testing was conducted in groups, on children from second to seventh grade. Children were then divided into a typical achievement (TA) group - no difficulties in the arithmetics and spelling subtests and a $\mathrm{MD}$ - score inferior to $1 \mathrm{SD}$ below the mean according to Brazilian norms in the arithmetics subtest.

Eighty-four typically achieving children (TA) and 14 children with MD took part in the individual neuropsychological testing phase. The two groups were matched regarding age and general intelligence. This sample was constituted by children with ages ranging from 8 to 11 years and normal intelligence $(z$-score between -1 and +1 in the Raven's Colored Progressive Matrices, see Table 1).

\section{INSTRUMENTS}

The following instruments were used in the neuropsychological assessment: Brazilian School Achievement Test (TDE; Stein, 1994), Raven's Colored Progressive Matrices, digit span (WISC), copy of Rey-Osterrieth Complex Figure, right-left orientation 
Table 1 | Descriptive data of the individual assessment sample.

\begin{tabular}{|c|c|c|c|c|c|c|c|}
\hline & \multicolumn{2}{|c|}{ TA } & \multicolumn{2}{|c|}{ MD } & $x^{2}$ & df & $p$ \\
\hline$N$ & \multicolumn{2}{|c|}{84} & \multicolumn{2}{|c|}{14} & & & \\
\hline Sex (\% female) & \multicolumn{2}{|c|}{64.3} & \multicolumn{2}{|c|}{57.1} & 0.263 & 1 & 0.608 \\
\hline Age (months) & 122.02 & 13.13 & 122.14 & 12.84 & -0.031 & 92 & 0.975 \\
\hline Raven ( $z$-score) & $\begin{array}{l}0.18 \\
(P R=57)\end{array}$ & $\begin{array}{l}0.49 \\
(\mathrm{Cl}=26-76)\end{array}$ & $\begin{array}{l}0.09 \\
(P R=54)\end{array}$ & $\begin{array}{l}0.67 \\
(\mathrm{Cl}=33-73)\end{array}$ & 0.576 & 96 & 0.566 \\
\hline
\end{tabular}

TA, typically achieving children; $M D$, children with mathematics difficulties; $P R$, percentile rank; Cl, confidence interval.

(Dellatolas et al., 1998), 9-hole peg test (Poole et al., 2005), handedness ascertainment (Lefèvre and Diament, 1982), non-symbolic magnitude comparison, symbolic magnitude comparison, finger localization test (Dellatolas et al., 1998), and tests for mathematical cognition (arithmetic word problems and basic arithmetic operations - addition, subtraction, and multiplication).

\section{Brazilian school achievement test (TDE; Stein, 1994)}

The TDE is the most widely used standardized test of school achievement with norms for the Brazilian population. It comprises three subtests: arithmetics, single-word spelling, and single-word reading. In the screening phase, we used the arithmetics and spelling subtests, which can be applied in groups. Norms are provided for school-aged children between the second and seventh grade. The arithmetics subtest is composed of three simple verbally presented word problems (i.e., which is the largest, 28 or 42 ?) and 45 written arithmetic calculations of increasing complexity (i.e., very easy: $4-1$; easy: $1230+150+1620$; intermediate: $823 \times 96$; hard: $3 / 4+2 / 8)$. Specific norms for each school grade were used to characterize children's individual performance. The spelling subtest consists of dictation of 34 words of increasing syllabic complexity (i.e., toca; balanço; cristalização). Reliability coefficients (Cronbach $\alpha$ ) of TDE subtests are 0.87 or higher. Children are instructed to work on the problems to the best of their capacity but without time limits.

\section{Raven's colored progressive matrices}

General intelligence was assessed with the age-appropriate Brazilian validated version of Raven's Colored Matrices (Angelini et al., 1999). Children with general intelligence below the 16 th percentile (i.e., $g<-1 \mathrm{SD}$ ) were not included in the sample.

\section{Digit span}

Verbal short-term memory was assessed with the Brazilian WISCIII Digits subtest (Figueiredo, 2002). Performance in the forward order was considered a measure of phonological short-term memory, and the backward order was used to assess verbal working memory.

\section{Corsi blocks (forward and backward)}

This test is a measure of the visuospatial component of working memory. It is constituted by a set of nine blocks which are tapped, in a certain sequence, by the examiner. The test starts with sequences of two blocks and can reach a maximum of nine blocks.
We used the forward and backward Corsi span tasks according to Kessels et al. (2000). In the forward condition, the child is instructed to tap the blocks on the same order as the examiner, in the backward condition, in the inverse order. Span is determined by the longest sequence correctly repeated before two successive failures.

\section{Rey-Osterrieth complex figure test (Strauss et al., 2006)}

The copy of the Rey figure assesses visuospatial and visuoconstructional abilities. It is based on a complex black and white drawing that the child must copy as accurately as possible. Accuracy score was based on 18 elements of the figure. For each correctly copied element children scored up to two points when the element was perfectly reproduced (Lezak et al., 2004; Strauss et al., 2006).

\section{Nine-hole peg test (9-HPT)}

The 9-HPT is a timed test in which nine pegs should be inserted and removed from nine holes in the pegboard with each hand; dominant and non-dominant. The version used was based on Poole et al. (2005). The pegboard is placed horizontally in front of the child, so that the compartment that contains the pegs is on the side of the hand to be tested, while the compartment with the holes is on its contralateral side. Children must pick one peg at a time. The test is performed two times with each hand, two consecutive attempts with the dominant hand, followed immediately by two consecutive attempts with the non-dominant hand. The scores were calculated based on the mean time for each hand.

\section{Handedness ascertainment}

Lateral preference was investigated by means of tasks that examine the ocular, hand, and foot preference based on Lefevre and Diament (1982). The child was instructed to look through a hole, to kick and to throw a ball, three times each. The result was given by the side the child had chosen more consistently.

\section{Right-left orientation test}

This test is based on Dellatolas et al. (1998). The test has 12 items of right and left body parts recognition. It is divided in three parts: the first part presents simple commands regarding the child's own body, the second consists of double commands - direct and crossed - toward the child's body. In the third part, pointing commands to single lateral body parts of an opposite-facing person were issued. Scores were attributed if the child correctly pointed to the nominated parts of the body; correct answers were coded 
with one and wrong answers with zero. Internal consistency was assessed with the Kuder-Richardson reliability coefficient, which was high $(\mathrm{KR}-20=0.80)$.

\section{Finger localization task}

This 24-item task also based on Dellatolas et al. (1998) was used to assess finger gnosia. It consists of three parts: (a) with the hand visible, localization of single fingers touched by the examiner with the pointed end of a pencil (two trials on each hand); (b) with the hand hidden from view, localization of single fingers touched by the examiner (four trials on each hand); (c) with the hand hidden from view, localization of pairs of fingers simultaneously touched by the examiner (six trials each hand). According to Dellatolas' et al. (1998) procedure, the participants were instructed to choose how they would rather respond: they could name the touched fingers, point to them on an outline drawing of the stimulated hand, or call out their numbers according to a figure in which fingers beginning with the thumb are numbered from 1 to 5 . A correct answer was coded 1 and a wrong answer 0 . A total score (ranging from 0 to 12 ) was calculated for each child. The internal consistency of this task is high $(\mathrm{KR}-20=0.79)$.

\section{Simple reaction time}

The computerized RT task is a visual detection task used to control for possible differences in basic processing speed, not related to numerical tasks. In this task the picture of a wolf (height $9.31 \mathrm{~cm}$; length $=11.59 \mathrm{~cm}$ ) was displayed in the center of a black screen for a maximum time of 3,000 ms. Participants were instructed to press the space bar on the keyboard as fast as possible whenever the wolf appeared. Each trial was terminated with the first key press. The task had 30 experimental trials, with an inter-trial interval varying between 2,000 and 8,000 ms.

\section{Non-symbolic magnitude comparison task}

In the non-symbolic magnitude comparison task, the participants were instructed to compare two simultaneously presented sets of dots, indicating which one contained the larger number. Black dots were presented on a white circle over a black background. On each trial, one of the two white circles contained 32 dots (reference numerosity) and the other one contained 20, 23, 26, 29, 35, 38,41 , or 44 dots. Each magnitude of dot sets was presented eight times. The task comprised 8 learning trials and 64 experimental trials. Perceptual variables were varied such that in half of the trials individual dot size was held constant, while in the other half the size of the area occupied by the dots was held constant (see exact procedure descriptions in Dehaene et al., 2005). Maximum stimulus presentation time was $4,000 \mathrm{~ms}$, and inter-trial interval was $700 \mathrm{~ms}$. Before each trial, a fixation point appeared on the screen - a cross, printed in white, with $30 \mathrm{~mm}$ in each line. If the child judged that the right circle presented more dots, a predefined key localized in the right side of the keyboard should be pressed with the right hand. On the contrary, if the child judged that the left circle contained more dots, then a predefined key on the left side had to be pressed with the left hand.

\section{Symbolic magnitude comparison task}

In the symbolic magnitude comparison task, Arabic digits from 1 to 9 were presented on the computer screen (height $=2.12 \mathrm{~cm}$; length $=2.12 \mathrm{~cm}$ ). The visual angle of the stimuli was $2.43^{\circ}$ in both vertical and horizontal dimensions. Children were instructed to compare the stimuli with the reference number 5 . Digits were presented in white on a black background. If the presented number was smaller than 5 , the child had to press a predefined key on the left side of the keyboard with the left hand. If the stimulus was larger than 5, the key to be pressed was located at the right side and should be pressed with the right hand. The number 5 was never presented. Numerical distances between stimuli and the reference number (5) varied from 1 to 4 , each numerical distance being presented the same number of times. Between trials a fixation point of the same size and color of the stimuli was presented on the screen. The task comprised 80 experimental trials. Maximum stimulus presentation time was $4,000 \mathrm{~ms}$, and inter-trial interval was $700 \mathrm{~ms}$.

\section{Arithmetic word problems}

Twelve arithmetical word problems were presented to the child on a sheet of paper while the examiner read them aloud simultaneously to avoid reading proficiency bias. There were six addition and six subtraction items, all of them with single-digit operands and results ranging from 2 to 9 (i.e., "Annelise has 9 cents. She gives 3 to Pedro. How many cents does Annelise have now?"). The child had to solve the problems mentally and write the answer down in Arabic format as quickly as possible, and the examiner registered the time taken for each item. Cronbach's $\alpha$ of this task was 0.83 .

\section{Basic arithmetic operations}

This task consisted of addition (27 items), subtraction (27 items), and multiplication (28 items) operations for individual application, which were printed on separated sheets of paper. Children were instructed to answer as fast and as accurate as they could, time limit per block being $1 \mathrm{~min}$. Arithmetic operations were organized in two levels of complexity and were presented to children in separated blocks: one consisted of simple arithmetic table facts and the other of more complex ones. Simple additions were defined as those operations with the results below 10 (i.e., $3+5$ ), while complex additions with the results between 11 and 17 (i.e., 9+5). Tie problems (i.e., 4+4) were not used for addition. Simple subtraction comprised problems in which the operands were below 10 (i.e., 9-6), while for complex subtractions the first operand ranged from 11 to 17 (i.e., $16-9)$. No negative results were included in the subtraction problems. Simple multiplication consisted of operations with results below 25 and with the number 5 as one of the operands (i.e., $2 \times 7,5 \times 6$ ), while for the complex multiplication the result of operands ranged from 24 to $72(6 \times 8)$. Tie problems were not used for multiplication. Reliability coefficients were high (Cronbach's $\alpha>0.90)$.

\section{ANALYSES}

Even though there was no statistical difference between groups regarding intelligence $(p=0.530)$, we decided to calculate the effect size of this difference $(d=0.182)$ and to include intelligence as a covariate in all further group comparisons. First, the differences between MD and TA groups regarding finger gnosia 
and other neuropsychological, cognitive, and numerical skills were investigated. Thereafter, the impact of finger gnosia on the differences between MD and TA groups in general cognitive functions (i.e., motor dexterity, right-left orientation) as well as numeric and arithmetic abilities (i.e., magnitude estimation, arithmetic word problems, simple and complex addition, subtraction, and multiplication problems) were examined. In a first set of ANCOVAS, group differences were calculated with intelligence as a covariate. In a second set of ANCOVAS, group differences were calculated again, but entering both intelligence and finger gnosia as covariates. Analyses in which finger gnosia reduced or even removed group differences were interpreted as indicative of a selective role of finger gnosia on specific cognitive functions. Finally, we examined the correlations of these variables. Finger discrimination was assessed separately for the left and the right hand. Only three children from the TA group and one MD were left-handed according to a lateralization test based on Lefèvre and Diament (1982). All analyses were duplicated excluding these children and the results did not differ so we did not exclude these individuals from statistical analyses. For this reason we considered finger gnosia for the left/right hand as an index of the non-dominant/ dominant hand.

\section{RESULTS}

We investigated differences in cognitive and numerical abilities between children with typical arithmetical abilities and children with MD in the following neuropsychological variables: the copy of Rey-Osterrieth complex figure test, digit span, Corsi Blocks,
9-HPT, finger gnosia, right-left orientation, arithmetic word problems, addition, subtraction, and multiplication operations, as well as symbolic and non-symbolic magnitude comparison tasks.

RT data was trimmed, eliminating in two steps all responses more extreme than 3 SD from the individual means, as well as those RTs faster than $200 \mathrm{~ms}$. Error data for the symbolic task was arcsine transformed to correct for skewness before entering statistical analysis. To analyze the non-symbolic task, we calculated for each children the internal Weber fraction (thereafter $w$ ), a measure previously used to estimate the acuity of the ANS (Piazza et al., 2004, 2010; Dehaene, 2007; Halberda et al., 2008; Izard and Dehaene, 2008; Mazzocco et al., 2011), based on the methods described by Piazza et al. (2004). One TA child refused to solve the subtraction task, and eight other TA children from early grades reported that they had not yet learned multiplication in school by the time of testing. Moreover, two TA and two MD did not complete the finger gnosia or the right-left orientation tasks, and nine TA did not complete the non-symbolic comparison task. Additionally, the $r^{2}$ of the fitting procedure to calculate the acuity of the ANS for three TA and for one MD children were less than $r^{2}=0.2$, so we did not consider the $w$ for those children. Furthermore, 11 TA children did not complete the symbolic magnitude comparison task. Table 2 describes the sample sizes, means and SD of the two groups, separately, for each measure.

A significant statistical difference between $\mathrm{MD}$ and TA was found for the finger gnosia task $[F(1,81)=9.04, \mathrm{MSE}=8.55$; $\left.p=0.004 ; \eta^{2}=0.10\right]$. When each hand was analyzed separately, the effect was much more pronounced for the left hand $[F$

Table 2 | Descriptive data of the neuropsychological measures for each group.

\begin{tabular}{|c|c|c|c|c|c|c|}
\hline \multirow[t]{2}{*}{ Tasks } & \multicolumn{2}{|c|}{$N$} & \multicolumn{2}{|c|}{ TA } & \multicolumn{2}{|c|}{ MD } \\
\hline & TA & MD & Mean & SD & Mean & SD \\
\hline Rey's figure (copy) & 84 & 14 & 27.33 & 6.43 & 27.96 & 4.77 \\
\hline Digits Wisc(forward) & 84 & 14 & 5.44 & 1.03 & 4.93 & 0.48 \\
\hline Digits Wisc(backward) & 84 & 14 & 3.21 & 0.91 & 2.79 & 0.43 \\
\hline Corsi blocks(forward) & 84 & 14 & 4.85 & 1.03 & 5.00 & 1.04 \\
\hline Corsi blocks (backward) & 84 & 14 & 4.17 & 0.97 & 4.36 & 1.01 \\
\hline 9-HPT(dominant hand)* & 72 & 12 & 20483.47 & 3174.69 & 20893.75 & 3773.14 \\
\hline 9-HPT(non-dominant hand)* & 72 & 12 & 21982.36 & 4511.07 & 22207.67 & 2499.94 \\
\hline Finger gnosia (right) & 72 & 12 & 10.38 & 1.74 & 9.33 & 2.27 \\
\hline Finger gnosia (left) & 72 & 12 & 10.71 & 1.56 & 8.83 & 2.59 \\
\hline Finger gnosia (both) & 72 & 12 & 21.08 & 2.79 & 18.17 & 4.47 \\
\hline Right/left orientation & 72 & 12 & 8.93 & 3.29 & 8.50 & 3.34 \\
\hline Arithmetics (TDE) & 84 & 14 & 18.32 & 6.16 & 10.93 & 4.79 \\
\hline Arithmetics word problems & 84 & 14 & 9.48 & 2.27 & 7.79 & 2.52 \\
\hline Addition & 84 & 14 & 10.76 & 2.99 & 9.64 & 3.03 \\
\hline Subtraction & 83 & 14 & 8.01 & 3.32 & 5.64 & 3.78 \\
\hline Multiplication & 76 & 14 & 8.26 & 4.47 & 3.39 & 2.76 \\
\hline Symbolic task_errors & 73 & 14 & 3.21 & 24.93 & 0.34 & 0.09 \\
\hline Symbolic task_RT* & 73 & 14 & 939.89 & 250.36 & 951.73 & 271.57 \\
\hline Non-symbolic task_RT** & 75 & 14 & 1262.98 & 351.81 & 1142.30 & 263.76 \\
\hline$W$ & 72 & 13 & 0.24 & 0.09 & 0.31 & 0.09 \\
\hline
\end{tabular}

TA, typically achieving children; MD, children with mathematics difficulties; 9-HPT, 9-hole peg test; RT, reaction time; W, Weber fraction; *time in milliseconds. 
$\left.(1,81)=11.56, \mathrm{MSE}=2.91 ; p=0.001 ; \eta^{2}=0.125\right]$ than for the right hand $\left[F(1,81)=3.02, \mathrm{MSE}=2.96 ; p=0.086 ; \eta^{2}=0.036\right]$. To investigate how finger gnosia is associated to other differences between TA and MD, two sets of ANCOVA models were calculated.

Statistical comparisons between groups revealed no significant differences between groups regarding the Rey-Osterrieth complex figure test, right-left orientation task, digit span (forward and backward), Corsi blocks (forward and backward), 9-HPT, simple reaction time task, symbolic and non-symbolic magnitude comparison tasks, and addition operations. In all of these comparisons, ANCOVA models including both intelligence and finger gnosia also remained non-significant (Table 3).

Significant differences between groups were found for the TDE, subtraction and multiplication operations, $w$, and arithmetic word problems. Importantly, after controlling for the impact of finger gnosia, all these comparisons remained significant, with the only exception of arithmetic word problems (Table 3 ).

Inspection of Table 4 reveals that the finger gnosia score correlated moderately with arithmetics subtest of the TDE and word problems. Moreover, all arithmetic tasks correlated moderately or strongly with each other. However, $w$ did not correlate with any other task rather than the arithmetics subtest of the TDE. Tasks tapping on core (the right-left orientation) as well as aggregated (visuospatial abilities, Rey-Osterrieth complex figure test) symptoms of the Gerstmann syndrome presented smaller correlations to finger gnosia compared to other tests that are not associated with the syndrome profile. Visuospatial working memory (Corsi
Blocks) also presented significant correlations to the arithmetics subtest of the TDE as well as to the basic arithmetic operations and to the arithmetic word problems (range $r=0.26$ to $r=0.41$ ).

\section{DISCUSSION}

In the present study the impact of finger gnosia on mathematics achievement was examined in a sample of children with and without MD. Finger gnosia performance is substantially lower in MD than in typically achieving (TA) children. This difference could not be attributed to general deficits in cognitive or somatomotor development, since these groups did not differ regarding general intelligence, working memory, visuospatial abilities or motor dexterity. After removing the effect of finger gnosia, the differences between MD and TA in arithmetic word problems disappeared. However, these differences remained significant in measures of mathematics achievement, acuity of the ANS (i.e., $w$ ) as well as in written subtraction and multiplication. In the following, these results will be discussed in more detail.

\section{GROUP DIFFERENCES IN FINGER GNOSIA}

In line with previous studies investigating the association between finger gnosia and numeric and arithmetic competencies in typically achieving children (Fayol et al., 1998; Noël, 2005; PennerWilger et al., 2007), the present study showed for the first time the existence of a deficit in finger gnosia in children selected for $\mathrm{MD}$ in comparison to typically developing children. Our results corroborate and extend the previous findings that the ability to discriminate fingers is specifically associated with numeric and

Table 3 | Analysis of covariance of the neuropsychological tasks (ANCOVA).

\begin{tabular}{|c|c|c|c|c|c|c|c|c|}
\hline \multirow[t]{2}{*}{ Tasks } & \multicolumn{4}{|c|}{$\begin{array}{c}\text { ANCOVA } \\
\text { (Covariate: intelligence) }\end{array}$} & \multicolumn{4}{|c|}{$\begin{array}{c}\text { ANCOVA } \\
\text { (Covariates: intelligence + gnosias) }\end{array}$} \\
\hline & $\boldsymbol{F}$ & df & $p$ & $\eta^{2}$ & $\boldsymbol{F}$ & df & $p$ & $\eta^{2}$ \\
\hline Rey's figure (copy) & 0.506 & $1 ; 95$ & 0.479 & 0.005 & 1.017 & $1 ; 80$ & 0.316 & 0.013 \\
\hline Digits Wisc(forward) & 3.119 & $1 ; 95$ & 0.081 & 0.032 & 2.477 & $1 ; 80$ & 0.119 & 0.030 \\
\hline Digits Wisc(backward) & 2.755 & $1 ; 95$ & 0.100 & 0.028 & 1.328 & $1 ; 80$ & 0.253 & 0.016 \\
\hline Corsi blocks (forward) & 0.421 & $1 ; 95$ & 0.518 & 0.004 & 2.025 & $1 ; 80$ & 0.159 & 0.025 \\
\hline Corsi blocks (backward) & 0.587 & $1 ; 95$ & 0.445 & 0.006 & 1.427 & $1 ; 80$ & 0.236 & 0.018 \\
\hline 9-HPT(dominant hand) & 0.051 & $1 ; 81$ & 0.822 & 0.001 & 0.053 & $1 ; 80$ & 0.818 & 0.001 \\
\hline 9-HPT(non-dominant hand) & 0.003 & $1 ; 81$ & 0.958 & $<0.001$ & 0.079 & $1 ; 80$ & 0.779 & 0.001 \\
\hline Right/left orientation & 0.067 & $1 ; 81$ & 0.796 & 0.001 & 0.001 & $1 ; 80$ & 0.970 & $<0.001$ \\
\hline Arithmetics (TDE) & 20.280 & $1 ; 95$ & $<0.001$ & 0.176 & 11.801 & $1 ; 80$ & 0.001 & 0.129 \\
\hline Arit. word problems & 6.496 & $1 ; 95$ & 0.012 & 0.064 & 1.467 & $1 ; 80$ & 0.229 & 0.018 \\
\hline Addition & 1.385 & $1 ; 95$ & 0.242 & 0.014 & 1.213 & $1 ; 80$ & 0.274 & 0.015 \\
\hline Subtraction & 5.655 & $1 ; 94$ & 0.019 & 0.057 & 4.831 & $1 ; 80$ & 0.031 & 0.057 \\
\hline Multiplication & 15.422 & $1 ; 87$ & $<0.001$ & 0.151 & 10.881 & $1 ; 74$ & 0.001 & 0.128 \\
\hline Simple reaction time & 2.915 & $1 ; 79$ & 0.092 & 0.036 & 0.604 & $1 ; 67$ & 0.440 & 0.009 \\
\hline Symbolic task_errors & 0.166 & $1 ; 85$ & 0.685 & 0.002 & 0.017 & $1 ; 72$ & 0.898 & $<0.001$ \\
\hline Symbolic task_RT & $<0.001$ & $1 ; 84$ & 1.000 & $<0.001$ & $<0.001$ & $1 ; 70$ & 0.992 & $<0.001$ \\
\hline Non-symbolic task_RT & 0.881 & $1 ; 86$ & 0.351 & 0.010 & 1.731 & $1 ; 73$ & 0.192 & 0.023 \\
\hline$W$ & 5.890 & $1 ; 82$ & 0.017 & 0.067 & 4.723 & $1 ; 70$ & 0.033 & 0.063 \\
\hline
\end{tabular}

Columns on the left show the set of ANCOVA models including only intelligence as a covariate. Columns on the right show those models including both intelligence and finger gnosia as covariates. TA, typically achieving children; MD, children with mathematics difficulties; 9-HPT, 9-hole peg test; RT, reaction time, W, Weber fraction. 


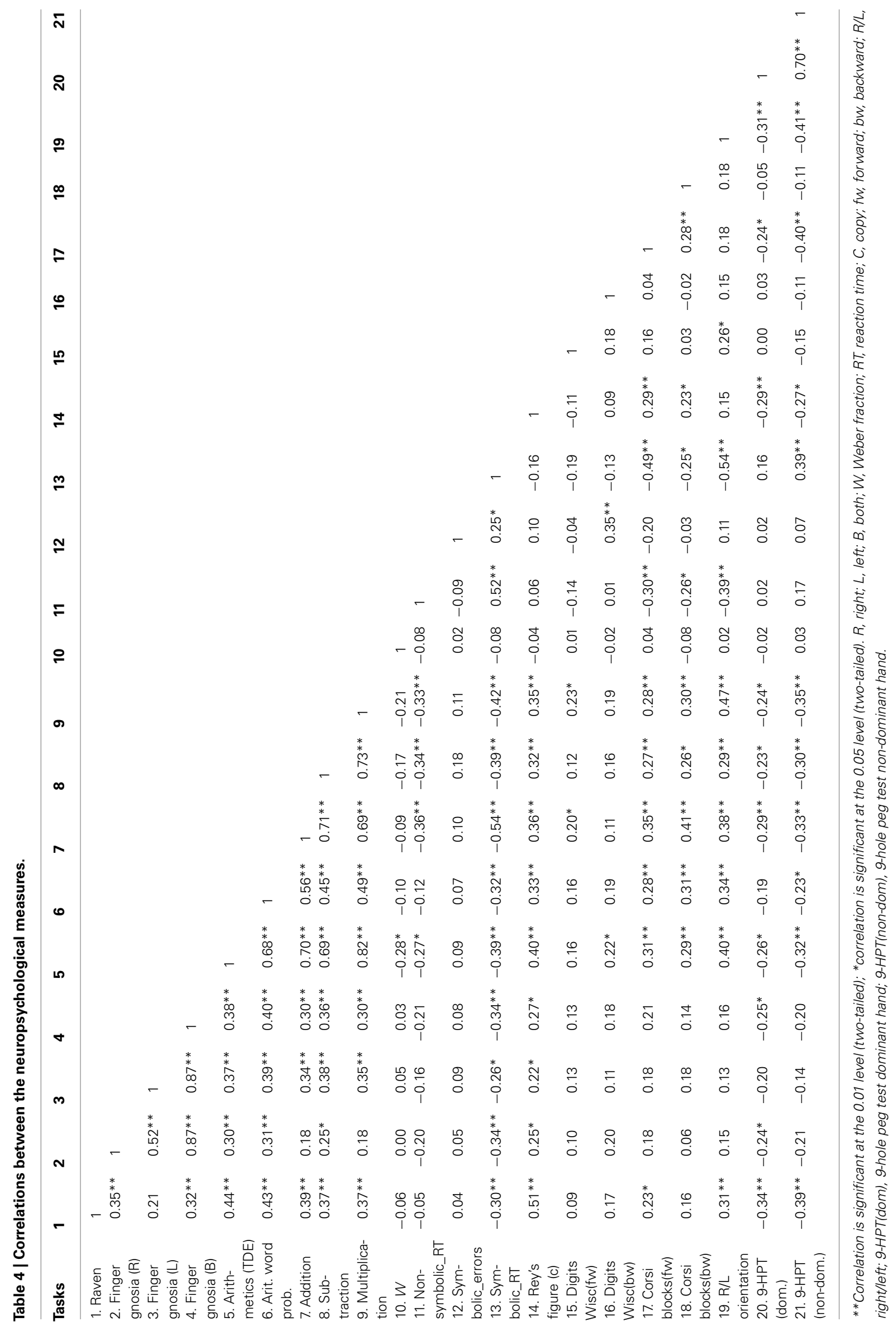


arithmetic abilities. Noël (2005) as well as Penner-Wilger et al. (2007) reported evidence that finger gnosia contributed independently to the prediction of numeric and arithmetic abilities in TA children. We found that finger gnosia discriminated between MD and TA even though general intelligence as well as verbal and non-verbal working memory were comparable across groups. Together, these studies suggest that finger gnosia contributes in a unique way to numeric and arithmetic abilities. Further evidence provided by Gracia-Bafalluy and Noël (2008) reinforces this conclusion. These authors have shown that children selected for their poor finger discrimination abilities performed worse in enumeration and counting tasks than children performing well in these number processing tasks. Altogether, these results are indicative that difficulties in MD children are not limited to basic magnitude representations but extend to finger representations. Finally, these results corroborate the view that the way finger discrimination determines numeric and arithmetic performance is related to working memory offloading (Alibali and DiRusso, 1999). As MD and TA children had comparable verbal and visuospatial working memory performance, MD may have failed to use finger representations to offload working memory and, for this reason, performed worse in arithmetic tasks intimately related to these capabilities. This topic will be discussed in the next section.

\section{DISENTANGLING FINGER GNOSIA FROM MORE GENERAL GROUP DIFFERENCES}

No differences in higher cognitive functions such as verbal and visuospatial working memory, visuoconstructional abilities, and general intelligence were found between MD and TA groups. In spite of the lack of differences between groups regarding these abilities, they all seem to have some peripheric relevance for the performance of arithmetical tasks, as can be seen in the small but significant correlations between them and arithmetic subtest of the TDE, arithmetic word problems, and basic arithmetic operations.

In summary, the deficit in finger discrimination observed in MD children does not seem to be related primarily to problems retaining and manipulating verbal or visuospatial information in a short-term buffer. Accordingly, if MD children may present difficulties offloading working memory (Alibali and DiRusso, 1999), these difficulties do not seem to be an outcome of capacity constraints or inability to apply the correct offloading strategy, since both MD and TA children showed comparable working memory capacity. Instead, the problems probably lay in accessing finger representations during calculation. Moreover, deficits in finger gnosia do not seem to be related to aspects of visuomotor transformations necessary for visuoconstructional abilities. This indicates that deficits in finger gnosia observed in MD children cannot be attributed to a generalized dysfunction of the left/right parietal lobes such as that observed for instance in Williams syndrome (Atkinson and Braddick, 2011) or in NLD more generally (Rourke, 1989). Finally, lack of difference in measures of general intelligence suggested that the deficit in finger gnosia is related to a very specific aspect of cognition and not to a general ability level. Therefore, one can be confident to explore a more specific link between finger gnosia and numeric and arithmetic abilities in the next section.

\section{FINGER GNOSIA AND NUMBER PROCESSING}

According to the functionalist hypothesis, finger gnosia should be related to some modalities of number processing and calculation but not to others. Finger gnosia should be relevant for tasks such as arithmetic word problems, which are frequently solved by beginning schoolers and MD children through finger counting. But finger gnosia should be less relevant for estimation or multiplication, which involve, respectively, magnitude estimation and fact retrieval (Noël, 2005). This prediction was corroborated by our empirical data.

Mathematical difficulties children presented higher $w$ compared to TA children. These results are consistent with ongoing literature showing a deficit in the acuity of the ANS in dyscalculic children as well as in children with mathematical learning disability (Piazza et al., 2010; Mazzocco et al., 2011). Furthermore, the mean values of $w$ found in this study $(\mathrm{TA}=0.23$ and $\mathrm{MD}=0.31)$ were very similar to the ones reported by Piazza et al., 2010; controls $=0.25$ and dyscalculics $=0.34$ ). More importantly, a deficit in such basic number magnitude discrimination seemed to be independent from a deficit encountered in finger gnosia in the present study. After removing the impact of finger gnosia abilities, the MD deficit in $w$ remained significant. Once more, these results suggest that finger gnosia provides a unique contribution to the deficits of MD children in numeric and arithmetic abilities. A controversial finding was the lack of group differences in the symbolic magnitude comparison task, which has also been reported in the literature regarding symbolic processing (onedigit magnitude comparison: Landerl et al., 2004; Mussolin et al., 2010).

In this study, MD performed worse than TA children in both multiplication and subtraction operation. After removing the impact of finger gnosia, these differences were not even attenuated. Despite the literature evidence concerning the relationship between finger gnosia and basic arithmetic operation (Ifrah, 2000; Crollen et al., 2011b), we did not find group differences specifically on the addition task. This might be due to the fact this task may have allowed for direct fact retrieval and be too simple for 10 or 11 years old children.

Noteworthy, after removing the effect of finger gnosia, the differences between MD and TA children observed in arithmetic word problems disappeared. Our results are in line with Butterworth's position, because they show that finger gnosia may serve as a mechanism to offload working memory demands, helping children to accurately represent quantities above the subitizing range, which in turn support arithmetic processing. According to Butterworth (1999), fingers a portable and always present tool used to link the abstract representation of numbers to concrete manipulations of quantities. As the capacity to perceive exact numerosities normally do not exceed four items, fingers are helpful to extend this limitation. Importantly, arithmetic word problems employed in the present study had solutions in the range between 2 and 9 . The usefulness of finger representations in numeric and arithmetic tasks seem to be limited to the number range encompassed between 1 and 10. When calculation problems exceed this interval, such as in our subtraction operations, the role of finger representations seems to fade. 
A final aspect of the result pattern obtained regards the specificity of the association between finger gnosia and numeric and arithmetic abilities. Previous studies have focused their attention on composite measures of these numeric and arithmetic abilities. Noël (2005) used a composite of comparison, Arabic digit comparison, subitizing, number writing, and addition as the dependent variable. Penner-Wilger et al. (2007) showed that finger gnosia was a predictor of number system knowledge, which was a summary variable consisting of digit recognition, counting, place value, and numeration. In the present study, the focus was more on the differential impact of finger gnosia on specific numeric and arithmetic tasks such as written subtraction and multiplication operations as well as word problems. Our results are, then, in accordance with the hypothesis that finger gnosia may play a role in certain arithmetic operations but not in others.

\section{FINGER GNOSIA, MATHEMATICS DIFFICULTIES, AND THE GERSTMANN SYNDROME}

Similarly to previous studies with neuropsychological patients (see review in Rusconi et al., 2010), the correlations between finger gnosia and other symptoms of the Gerstmann syndrome such as right-left orientation or aggregated visuospatial abilities were weak. In contrast, the correlation between finger gnosia and other numeric and arithmetic abilities tended to be higher. Together, these results are indicative that the deficits presented by MD children have another cause than a Gerstmann-like kind of disorder and are in accordance with previous findings (Rusconi et al., 2009).

Another source of evidence lending support to this conclusion is that finger gnosia deficits in MD children were more pronounced for the left hand than for the right hand. While the Gerstmann syndrome is typically associated with parietal lesions in the hemisphere dominant for language, more pronounced effects of finger gnosia were observed for the non-dominant left hand. Further evidence from neuropsychology as well as structural and functional imaging studies also supports the larger involvement of the non-dominant left hand in finger agnosia. A preponderance of left hand difficulties in sensorimotor and body perception in children with NLD (non-verbal learning disability) was observed by Rourke (summarized by Rourke, 1989). According to Rourke (1989, 1995), preponderant right hemisphere expression of symptoms in NLD may be explained by white matter subcortical dysfunctions, as observed in several genetic syndromes related to the disorder such as Turner and 22q11.2 deletion syndrome (Barnea-Goraly et al., 2003; Molko et al., 2004). Structural imaging techniques of cortical white matter have recently renewed interest in disconnection interpretations of several disorders, including learning disorders (Mitchell, 2011). Some results also point to interhemispheric asymmetries. Rykhlevskaia et al. (2009) observed that, besides gray matter alterations in relevant parietal cortical regions, underlying white matter fractional anisotropy and volume alterations on the posterior right hemisphere were also associated to developmental dyscalculia. Moreover, a hemispheric asymmetry related to magnitude processing in MD children, which indicated a malfunction of the right parietal cortex, has been observed by Kaufmann et al. (2009). In summary, neuropsychologic and imaging studies are suggestive about the existence of a functional link between right parietal functions, finger gnosia in the non-dominant hand, and numeric and arithmetic abilities.

\section{PRACTICAL IMPLICATIONS}

Specific deficits in finger gnosia observed in MD children are indicative that measures of finger gnosia may be useful in the early screening of MD (Noël, 2005). Research efforts are currently directed toward identifying cognitive/behavioral markers that could be easily employed by teachers in the identification of children at risk of developing MD (Mazzocco and Thompson, 2005; Geary et al., 2009; LeFevre et al., 2010). Evidence found in the present study advanced the discussion on the search after markers in two different ways. First, finger gnosia abilities differ substantially between MD and TA children. This suggests that finger gnosia can be used as a marker of MD. As reviewed by Beller and Bender (2011), finger abilities are not indispensable for the development of numerical competence, but they could increase children's numerical performance. Moreover, the absence of correlation between $w$ and finger gnosia suggest that finger gnosia and acuity of the ANS could be dissociable. Therefore, finger gnosia seems to make an independent contribution to MD.

Research should focus on the relative predictive power of finger gnosia, adding measures of finger gnosia to the characterization of basic numeric abilities. Further studies should also investigate calculation strategies, specifically the use of overt finger representation. Finally, future studies investigating how finger discrimination training may be improved could be a valuable tool in the prevention and rehabilitation of MD (Gracia-Bafalluy and Noël, 2008). As it already occurs in some countries (Guha, 2006), finger discrimination training could be introduced explicitly in the preschool curriculum, thereby helping children to establish a functional bridge between magnitudes and their symbolic representations, facilitating calculation procedures.

In summary, deficits in finger gnosia are associated to MD in 8- to 11-years-old children. Finger gnosia seems to be particularly relevant for the performance in word problems requiring active manipulation of small magnitudes in the range between 1 and 10. Importantly, evidence relating finger gnosia to more complex calculations in a range of magnitudes over 10 was not found. Moreover, the deficits in finger gnosia could not be attributed to a shortage in working memory capacity but rather to a specific inability to use fingers to transiently represent magnitudes, tagging to be counted objects, and thereby reducing the cognitive load necessary to solve arithmetic problems. Since finger gnosia was more related to symbolic than to non-symbolic magnitude processing in our study, finger-related representation of magnitude seems to be an important link for learning the mapping of analog onto discrete symbolic magnitudes.

\section{ACKNOWLEDGMENTS}

Research by the Vitor Geraldi Haase during the elaboration of this article was funded by grants from CAPES/DAAD Probral Program, Conselho Nacional de Desenvolvimento Científico e Tecnológico (CNPq, 307006/2008-5, 401232/2009-3), and Fundação de Amparo à Pesquisa do Estado de Minas Gerais (FAPEMIG, APQ-02755-SHA, APQ-03289-10. Guilherme Wood is supported by a FWF research project (nr. P22577). 


\section{REFERENCES}

Alibali, M., and DiRusso, A. (1999). The function of gesture in learning to count: more than keeping track. Cogn. Dev. 14, 37-56.

Andres, M., Seron, X., and Olivier, E. (2007). Contribution of hand motor circuits to counting. J. Cogn. Neurosci. 19, 563-576.

Angelini, A. L., Alves, I. C. B., Custódio, E. M., Duarte, W. F., and Duarte, J. L. M. (1999). Matrizes progressivas coloridas de Raven - escala especial. São Paulo: Centro Editor de Testes e Pesquisas em Psicologia.

Atkinson, J., and Braddick, O. (2011). From genes to brain development to phenotypic behavior: "dorsalstream vulnerability" in relation to spatial cognition, attention, and planning of actions in Williams syndrome (WS) and other developmental disorders. Prog. Brain Res. 189, 261-283.

Barnea-Goraly, N., Menon, V., Krasnow, B., Ko, A., Reiss, A., and Eliez, S. (2003). Investigation of white matter structure in velocardiofacial syndrome: a diffusion tensor imaging study. Am. Psychiatr. Assoc. 160, 1863-1869.

Beller, S., and Bender, A. (2011). Explicating numerical information: when and how fingers support (or hinder) number comprehension and handling. Front. Psychol. 2:214. doi:10.3389/fpsyg.2011.00214

Bull, R., and Scerif, G. (2001). Executive functioning as a predictor of children's mathematics ability: inhibition, switching, and working memory. Dev. Neuropsychol. 19, 273-293.

Butterworth, B. (1999). What Counts: How Every Brain is Hardwired for Math. New York: The Free Press.

Conson, M., Mazzarella, E., and Trojano, L. (2009). Numbers are represented in egocentric space: effects of numerical cues and spatial reference frames on hand laterality judgments. Neurosci. Lett. 452, 176-180.

Crollen, V., Mahe, R., Collignon, O., and Seron, X. (2011a). The role of vision in the development of finger-number interactions: fingercounting and finger-montring in blind children. J. Exp. Child Psychol. 109, 525-539.

Crollen, V., Seron, X., and Noël, M. P. (2011b). Is finger-counting necessary for the development of arithmetic abilities? Front. Psychol. 2:242. doi:10.3389/fpsyg.2011.00242

Dehaene, S. (2007). "Symbols and quantities in parietal cortex: elements of a mathematical theory of number representation and manipulation," in Sensorimotor Foundations of Higher Cognition - Attention and Performance XXII, eds P. Haggard, Y. Rossetti, and M. Kawato (Cambridge, MA: Harvard University Press), 527-574.

Dehaene, S., and Cohen, L. (2007). Cultural recycling of cortical maps. Neuron 56, 384-398.

Dehaene, S., Izard, I., and Piazza, M. (2005). Control Over Non-Numerical Parameters in Numerosity Experiments. Available at: www.unicog. org/docs/DocumentationDotsGene ration.doc

Dellatolas, G., Viguier, D., Deloche, G., and De Agostini, M. (1998). Rightleft orientation and significance of systematic reversal in children. Cortex 34, 659-676.

Di Luca, S., Granà, A., Semenza, C., Seron, X., and Pesenti, M. (2010). Finger-digit compatibility in Arabic numeral processing. Q. J. Exp. Psychol. 59, 1648-1663.

Domahs, F., Krinzinger, H., and Willmes, K. (2008). Mind the gap between both hands: evidence for internal finger-based number representations in children's mental calculation. Cortex 44, 359-367.

Domahs, F., Moeller, K., Huber, S., Willmes, K., and Nuerk, H. C. (2010). Embodied numerosity: implicit hand-based representations influence symbolic number processing across cultures. Cognition 116, 251-266.

Fayol, M., Barrouillet, P., and Marinthe, C. (1998). Predicting arithmetic achievement from neuropsychological performance: a longitudinal study. Cognition 68, 63-70.

Figueiredo, V. L. M. (2002). WISCIII: Escala de Inteligência Wechsler para Crianças. Manual Adaptação e Padronização Brasileira. São Paulo: Casa do Psicólogo.

Fischer, J. P. (2010). Numerical performance increased by finger training: a fallacy due to regression toward the mean? Cortex 46, 272-273.

Fischer, M. H. (2008). Finger counting habits modulate spatial-numerical associations. Cortex 44, 386-392.

Geary, D. C., Bailey, D. H., and Hoard, M. K. (2009). Predicting mathematical achievement and mathematical learning disability with a simple screening tool: the number sets test. J. Psychoeduc. Assess. 27, 265-279.

Geary, D. C., Hamson, C. O., and Hoard, M. K. (2000). Numerical and arithmetical cognition: a longitudinal study of process and concept deficits in children with learning disability. J. Exp. Child Psychol. 77, 236-263.

Gerstmann, J. (1940). Syndrome of finger gnosia, disorientation for left and right, dysgraphia and acalculia. Arch. Neurol. Psychiatry 44, 398-408.

Gracia-Bafalluy, M., and Noël, M. P. (2008). Does finger training increase young children's numerical performance? Cortex 44, 368-375.

Guha, S. (2006). Using mathematics strategies in early childhood education as a basis for culturally responsive teaching in India. Int. J. Early Years Educ. 14, 15-34.

Halberda, J., Mazzocco, M. M. M., and Feigenson, L. (2008). Individual differences in non-verbal number acuity correlate with maths achievement. Nature 455, 665-668.

Hecht, S. A., Torgesen, J. K., Wagner, R. K., and Rashotte, C. A. (2001). The relations between phonological processing abilities and emerging individual differences in mathematical computation skills: a longitudinal study from second to fifth grades. J. Exp. Child Psychol. 79, 192-227.

Ifrah, G. (2000). The Universal History of Numbers. London: Harvill.

Izard, V., and Dehaene, S. (2008). Calibrating the mental number line. Cognition 106, 1221-1247.

Jordan, N. C., Kaplan, D., Ramineni, C., and Locuniak, M. N. (2008). Development of number combination skill in the early school years: when do fingers help? Dev. Sci. 11, 661-668.

Kaufmann, L., Vogel, S. E., Starke, M., Kremser, C., Schocke, M., and Wood, G. (2009). Developmental dyscalculia: compensatory mechanisms in left intraparietal regions in response to nonsymbolic magnitudes. Behav. Brain Funct. 5, 5-35.

Kaufmann, L., Vogel, S. E., Wood, G., Kremser, C., Schocke, M., Zimmerhackl, L. B., and Koten, J. W. (2008). A developmental fMRI study of nonsymbolic numerical and spatial processing. Cortex 44, 376-385.

Kaufmann, L., Wood, G., Rubinsten, O., and Henik, A. (2011). Metaanalyses of developmental fMRI studies investigating typical and atypical trajectories of number processing and calculation. Dev. Neuropsychol. 36, 763-787.

Kessels, R. P. C., van Zandvoort, M. J. E., Postma, A., Kapelle, L. J., and Haan, E. H. F. (2000). The Corsi blocktapping task: standardization and normative data. Appl. Neuropsychol. 7, 252-258.

Kinsbourne, M., and Warrington, E. K. (1963). The developmental Gerstmann syndrome. Ann. Neurol. 8, 490-501.

Landerl, K., Bevan, A., and Butterworth, B. (2004). Developmental dyscalculia and basic numerical capacities: a study of 8-9-years-old students. Cognition 93, 99-125.

Lecointre, A. S., Lépine, R., and Camos, V. (2005). "Développement et troubles des processus de quantification," in La Dyscalculie Trouble du Développment Numérique de L'enfant, ed. M. P. Noël (Marseille: SOLAL), 41-75.

Lefèvre, A. B., and Diament, A. J. (1982). Epidemiologia em neurologia infantil: estudo dos diagnósticos mais comuns. Revista do Hospital das Clínicas Faculdade de Médica de São Paulo 37, 199-205.

LeFevre, J. A., Fast, L., Skwarchuk, S. L., Smith-Chant, B. L., Bisanz, J., Kamawar, D., and PennerWilger, M. (2010). Pathways to mathematics: longitudinal predictors of performance. Child Dev. 81, 1753-1767.

Lezak, M. D., Howieson, D. B., and Loring, D. W. (2004). Neuropsychological Assessment. New York: Oxford University Press.

MacCallum, R. C., Widaman, K. F., Zhang, S., and Hong, S. (1999). Sample size in factor analysis. Psychol. Methods 4, 84-99.

Marinthe, C., Fayol, M., and Barrouillet, P. (2001). "Gnosies digitales et developpement des performances arithmetiques," in Troubles du Calcul et Dyscalculies chez L'enfant, eds A. Van Hout and C. Meljac (Paris: Masson), 239-254.

Mazzocco, M. M. M., Feigenson, L., and Halberda, J. (2011). Impaired acuity of the approximate number system underlies mathematical learning disability (dyscalculia). Child Dev. 82, 1224-1237.

Mazzocco, M. M. M., and Thompson, R. E. (2005). Kindergarten predictors of math learning disability. Learn. Disabil. Res. Pract. 20, 142-155.

Mitchell, K. J. (2011). Curiouser and curiouser: genetic disorders of cortical specialization. Curr. Opin. Genet. Dev. 21, 197-203

Molko, N., Cachia, A., Riviere, D., Mangin, J. F., Bruandet, M., LeBihan, D., Cohen, L., and Dehaene, S. (2004). Brain anatomy in Turner syndrome: evidence for impaired social and spatial-numerical networks. Cereb. Cortex 14, 840-850.

Mussolin, C., Mejias, S., and Noël, M. P. (2010). Symbolic and nonsymbolic number comparison in children with and without dyscalculia. Cognition 115, 10-25.

Noël, M. P. (2005). Finger gnosia: a predictor of numerical abilities in children? Child Neuropsychol. 11, 413-430. 
Penner-Wilger, M., and Anderson, M. L. (2008). "An alternative view of the relation between finger gnosis and math ability: redeployment of finger representations for the representation of numbers," in Proceedings of the 30th Annual Meeting of the Cognitive Science Society, eds B. C. Love, K. McRae, and V. M. Sloutsky (Austin, TX: Cognitive Science Society), 1647-1652.

Penner-Wilger, M., Fast, L., LeFèvre, J., Smith-Chant, B. L., Skwarchuk, S., Kamawar, D., and Bisanz, J. (2007). "The foundations of numeracy: subitizing, finger gnosia, and fine-motor ability," in Proceedings of the 29th Annual Cognitive Science Society, eds D. S. McNamara and J. G. Trafton (Austin, TX: Cognitive Science Society), 1385-1390.

Piazza, M., Facoetti, A., Trussardi, A. N., Berteletti, I., Conte, S., Lucangeli, D., Dehaene, S., and Zorzi, M. (2010). Developmental trajectory of number acuity reveals a severe impairment in developmental dyscalculia. Cognition 116, 33-41.

Piazza, M., Izard, V., Pinel, P., Le Bihan, D., and Dehaene, S. (2004). Tuning curves for approximate numerosity in the human intraparietal sulcus. Neuron 44, 547-555.

Poole, J. L., Burtner, P. A., Torres, T. A., McMullen, C. K., Markham, A., Marcum, M. L., Anderson, J. B., and Qualls, C. (2005). Measuring dexterity in children using the ninehole peg test. J. Hand Ther. 18, 348-351.

Raghubar, K. P., Barnes, M. A., and Hecht, S. A. (2010). Working memory and mathematics: a review of developmental, individual difference, and cognitive approaches. Learn. Individ. Differ. 20, 110-122.

Rourke, B. (1995). Syndrome of Nonverbal Learning Disability: Neurodevelopmental Manifestations. New York: Gilford Press.

Rourke, B. P. (1989). Nonverbal Learning Disability: the Syndrome and the Model. New York: Guilford.

Rusconi, E., Pinel, P., Dehaene, S., and Kleinschmidt, A. (2010). The enigma of Gerstmann's syndrome revisited: a telling tale of the vicissitudes of neuropsychology. Brain 133, 320-332.

Rusconi, E., Pinel, P., Eger, E., LeBihan, D., Thirion, B., Dehaene, S., and Kleinschmidt, A. (2009). A disconnection account of Gerstmann syndrome: functional neuroanatomy evidence. Ann. Neurol. 66, 654-662.

Rykhlevskaia, E., Uddin, L. Q., Kondos, L., and Menon, V. (2009). Neuroanatomical correlates of developmental dyscalculia: combined evidence from morphometry and tractography. Front. Hum. Neurosci. 3:51. doi:10.3389/neuro.09.051.2009

Sato, M., Cattaneo, L., Rizzolatti, G., and Gallese, V. (2007). Numbers within our hands: modulation of corticospinal excitability of hand muscles during numerical judgment. J. Cogn. Neurosci. 19, 684-693.

Stein, L. M. (1994). TDE - Teste de Desempenho Escolar. Manual para aplicação e interpretação. São Paulo: Casa do Psicólogo.

Strauss, E., Sherman, E. M. S., and Spreen, O. (2006). A Compendium of Neuropsychological Tests: Administration, Norms, and Commentary. New York: Oxford University Press.

Swanson, H. L. (2004). Working memory and phonological processing as predictors of children's mathematical problem solving at different ages. Mem. Cognit. 32, 648-661.

van der Sluis, S., de Jong, P. F., and van der Leij, A. (2004). Inhibi- tion and shifting in children with learning deficits in arithmetic and reading. J. Exp. Child Psychol. 87, 239-266.

Conflict of Interest Statement: The authors declare that the research was conducted in the absence of any commercial or financial relationships that could be construed as a potential conflict of interest.

Received: 30 July 2011; accepted: 23 November 2011; published online: 12 December 2011.

Citation: Costa AJ, Silva JBL, Chagas PP, Krinzinger H, Lonneman J, Willmes $K$, Wood G and Haase VG (2011) A hand full of numbers: a role for offloading in arithmetics learning? Front. Psychology 2:368. doi: 10.3389/fpsyg.2011.00368

This article was submitted to Frontiers in Cognition, a specialty of Frontiers in Psychology.

Copyright (c) 2011 Costa, Silva, Chagas, Krinzinger, Lonneman, Willmes, Wood and Haase. This is an open-access article distributed under the terms of the Creative Commons Attribution Non Commercial License, which permits noncommercial use, distribution, and reproduction in other forums, provided the original authors and source are credited. 